\title{
Distributed Turbo Coding Scheme with Punctured Soft Systematic-bit Repeat in Relay Systems over Quasi-static Rayleigh Fading Channels
}

\author{
Muzi Wu, Yuexing Peng, Wenbo Wang \\ Wireless Signal Processing and Network Laboratory \\ Key Lab of Universal Wireless Communication, Ministry of Education \\ School of Information and Communication Engineering \\ Beijing University of Posts \& Telecommunications, Beijing, China
}

\begin{abstract}
In order to mitigate the error propagation and achieve diversity gain, an enhanced distributed turbo coding (DTC) with soft information relaying (SIR) scheme is proposed for relay systems in practical fading channels. For the proposed scheme, not only the soft information of the additional parity bits but also the soft information of the systematic bits are regenerated and repeated at the relay. To avoid the penalty of spectrum efficiency loss due to the repeat of information on systematic bits, puncturing is employed. Bit error probability performance of the proposed DTC-SIR scheme is analyzed via distance spectrum and union bound method. Both the theoretic analysis and the numerical simulation show that the proposed scheme outperforms the traditional DTC scheme in practical fading channels.
\end{abstract}

Keywords-relay; distributed turbo coding; systematic-bit repeat

\section{INTRODUCTION}

The basic idea of relay-based wireless networks is that one terminal transmits signals to another through a number of relays. The relays not only receive and route the incoming signals, but also make some operations on the received signals before forward them. The introductions of one or multiple relays offer better communication efficiency beyond the single link communication paradigm. The relay-based networks offer tremendous advantages such as the source can simply but effectively leverage the processing and transmit power, and the intrinsic spatial diversity can be implemented by the use of the relay. In this paper, the typical relay system with three-node model is concerned, which consists of a source $(S)$, a relay $(R)$ and a destination (D), as depicted in Fig. 1. In view of easy implementation, time-division half-duplex is assumed for the system, which means that the relay receives and transmits in different time slot.

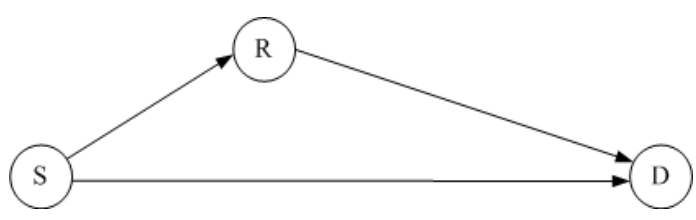

Fig. 1. Block diagram of the simplest relay-based network.
The essential study of the relay-based networks in physical layer is how to design the signal relaying strategies to achieve the best system performance. Two classical strategies are proposed in [1], namely, amplify-forward (AF) and decode-forward (DF). Amplify-forward is straight-forward, requiring a lower implementation complexity in digital signal processing, where the relay scales, retransmits or reflects the analog signal waveforms received from the source [2]. However, AF scheme forwards the reliability information extracted directly from the transmit channel without utilizing the channel code that may well exist in the source's information sequences [3]. On the other hand, DF utilizes the gain provided by channel code at the relay, where signals from the source are decoded and possibly re-encoded [2]. DF exploits the coding gain but forwards the hard-decision information which may result in decoding error propagation due to the coherent error-prone feature of the fading channels. These observations motivate an efficient relaying scheme, decode-amplify-forward, which combines the merits of AF and DF, by having the relay perform soft decoding and forward the soft information of the decoded bits instead of that from the raw channel as AF [3].

The idea of using channel code as DF opens the possibility for maximizing the coding gain in a relay network, some distributed coding schemes [4]-[8] have been developed recently. Here, we focus on traditional distributed turbo coding (DTC) scheme which offers better coding gains and performs close to the theoretic outage probability bound of a relay channel [7][8]. DTC scheme with half-duplex transmit modes is operated in two stages. In the first stage, the source broadcasts the coded signals to both the relay and the destination. In the second stage, the relay decodes, interleaves and re-encodes the information prior to forwarding. The coded signals received from the source and the relay in successive two stages naturally constitute two inputs of turbo decoder, so the turbo decoding can be implemented at the destination.

When error-free decoding is performed at the relay, the traditional DTC scheme approaches the information-theoretic capacity of relay channels. However, decoding error is inevitable in practical fading channels, and consequently error propagation is introduced when the decoding error is forwarded by the traditional DTC scheme. Decoding error propagation effect severely degrades the performance of DTC scheme. To 
mitigate it, distributed turbo coding with soft information relaying (DTC-SIR) scheme is proposed in [8], where the relay uses soft input soft output (SISO) decoder and SISO re-encoder, and then forwards the soft information of the re-encoded additional parity bits. Moreover, it has also been shown in [9] that forwarding the additional parity bits as well as the repeating systematic bits can improve the system performance due to the diversity improvement in a block fading channel.

Inspired by the method in [9] and rooted in the recent development of DTC-SIR scheme, we propose an enhanced DTC-SIR scheme to mitigate the decoding error propagation and gain diversity as well for relay-enhanced wireless communication systems over practical fading channels. For the proposed scheme, the relay forwards the soft information of both the systematic bits and the additional parity bits. In order to avoid the penalty of spectrum efficiency loss due to the repetition of the systematic bits and to keep the same cooperation level as traditional DTC scheme, part of the soft information of the systematic bits and the additional parity bits are punctured. Numerical simulation is implemented to evaluate the proposed DTC scheme in practical fading channels. The simulation results show that the proposed scheme outperforms traditional DTC scheme in [7] and DTC-SIR scheme in [8].

\section{SYSTEM MODEL}

As stated above, time-division half-duplex is assumed for the concerned relay system. The proposed scheme operates in two time slots. In the first time slot, the information bit stream of length $K$ is encoded into a codeword with code rate $R$. The recursive systematic convolutional (RSC) coding is employed in this paper. The codeword is mapped into constellation signal sequences. For simplicity and without loss of generality, binary phase-shift keying (BPSK) modulation is assumed. The first frame consists of the modulated symbol sequence, which is broadcasted to both the destination and the relay. The block diagram of the processing at the source is depicted in Fig. 2.

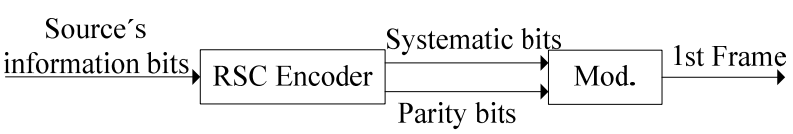

Fig. 2. The block diagram of the processing at the source.

The received signals at the relay and destination, denoted by $\mathbf{y}_{\mathrm{SR}}$ and $\mathbf{y}_{\mathrm{SD}}$, respectively, can be expressed as

$$
\begin{aligned}
& \mathbf{y}_{\mathrm{SR}}=h_{\mathrm{SR}} \mathbf{x}_{\mathrm{S}}+\mathbf{n}_{\mathrm{SR}} \\
& \mathbf{y}_{\mathrm{SD}}=h_{\mathrm{SD}} \mathbf{x}_{\mathrm{S}}+\mathbf{n}_{\mathrm{SD}}
\end{aligned}
$$

where $\mathbf{x}_{\mathrm{S}}$ is the modulated symbol sequence from the source, $h_{\mathrm{SR}}$ and $h_{\mathrm{SD}}$ are the fading coefficients of the source-to-relay channel and the source-to-destination channel, respectively. They are modeled as zero-mean, independent circularly symmetric complex Gaussian random variables with variance $1 / 2$ per dimension. In this paper, quasi-static fading channel is assumed, and the fading coefficients are constant within one frame but change independently from one frame to another. The transmitted signals are also disturbed by the additive white Gaussian noises, $\mathbf{n}_{\mathrm{SR}}$ and $\mathbf{n}_{\mathrm{SD}}$, which are assumed to be zero mean and Gaussian random variables with variances $\sigma_{\mathrm{SR}}^{2}$ and $\sigma_{\mathrm{SD}}^{2}$, respectively. The signal to noise ratio (SNR) is $E\left\{h_{l}^{2}\right\} E_{\mathrm{S}} / \sigma_{l}^{2}, l \in\{S R, S D\}$, where $E_{\mathrm{S}}$ is the average symbol power and is assumed to be unity.

In the second time slot, the relay processes the signals from the source, and forwards the second frame consisted of the signals $\mathbf{x}_{R}$. The signal received at the destination is

$$
\mathbf{y}_{\mathrm{RD}}=h_{\mathrm{RD}} \mathbf{x}_{\mathrm{R}}+\mathbf{n}_{\mathrm{RD}}
$$

where $h_{\mathrm{RD}}$ is the fading coefficient of the quasi-static fading channel between the relay and the destination, $\mathbf{n}_{\mathrm{RD}}$ is the zero mean and Gaussian random variables with variances $\sigma_{\mathrm{RD}}^{2}$.

\section{The Proposed ENHANCED DTC-SIR SCHEME}

\section{A. Coding scheme}

The processing at the relay is depicted in Fig. 3. After receiving the signals from the source, the relay demodulates and decodes the signals by SISO decoder with maximum a posteriori probability (MAP) decoding algorithm to calculate the soft information of the systematic bits in the form of log-likelihood ratios (LLRs), denoted by $L_{\text {systematic. The soft }}$ information of the systematic bits are interleaved, and then fed to the SISO re-encoder to produce the soft information of the additional parity bits, denoted by $L_{\text {parity. }}$. Detailed discussions on the SISO re-encoder can be found in [8].

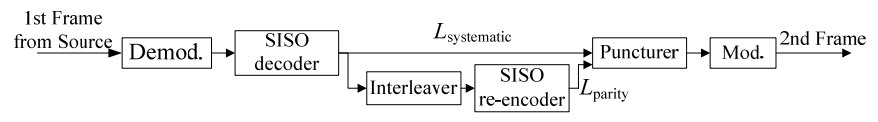

Fig. 3. The block diagram of the processing at the relay.

Different from the previous DTC scheme with hard information forwarding (HIR) or soft information forwarding (SIR), the proposed DTC scheme forwards the soft information of the systematic bits besides the soft information of the additional parity bits both in the form of LLRs. Puncturing scheme is used to keep the same cooperation level as traditional DTC scheme and to avoid the loss of spectrum efficiency due to the systematic information repeating. For example, half soft systematic bits and half soft additional parity bits are punctured to assure the same data rate as conventional DTC-SIR scheme. Then, the punctured soft information sequences are mapped to the transmitted symbol sequence using BPSK with the same bandwidth efficiency as the traditional DTC.

Given BPSK modulation scheme, the binary bit 0 or 1 is mapped into 1 or -1 . For the proposed DTC scheme, since the soft information is in the form of LLR, the mapping of the soft information is performed as follows.

Assuming $L_{\mathrm{R}}(k)$ is the soft information at the relay, where $k=1,2, \ldots, L$, and $L$ is the second frame size.

$$
L_{\mathrm{R}}(k)=\log \frac{p\left(\tilde{c}(k)=0 \mid \mathbf{y}_{\mathrm{SR}}\right)}{p\left(\tilde{c}(k)=1 \mid \mathbf{y}_{\mathrm{SR}}\right)}
$$


The probability of the binary symbol is

$$
\begin{aligned}
\hat{c}(k) & =p\left(\tilde{c}(k)=1 \mid \mathbf{y}_{\mathrm{SR}}\right) \cdot 1+p\left(\tilde{c}(k)=0 \mid \mathbf{y}_{\mathrm{SR}}\right) \cdot 0 \\
& =p\left(\tilde{c}(k)=1 \mid \mathbf{y}_{\mathrm{SR}}\right)
\end{aligned}
$$

So $\hat{c}(k)$ can be modulated as follows:

$$
\begin{aligned}
x_{\mathrm{R}}(k) & =1-2 \hat{c}(k) \\
& =1-\frac{2}{1+\exp \left(L_{\mathrm{R}}(k)\right)} \\
& =\tanh \left(\frac{L_{\mathrm{R}}(k)}{2}\right)
\end{aligned}
$$

$\mathbf{x}_{\mathrm{R}}$ is then forwarded to the destination.

\section{B. Decoding scheme}

The decoding processing at the destination is depicted in Fig. 4. The received signals $\mathbf{y}_{\mathrm{SD}}, \mathbf{y}_{\mathrm{RD}}$ from the source and the relay are first demodulated to obtain the soft channel information. Assuming channel state information is available at the destination, each demodulator first computes the LLRs of all the coded bits. Since both the signals from the source and the relay contain the information of the systematic bits, soft combining is performed, i.e., maximum ratio combining (MRC). The LLRs are split into three streams pertaining to the systematic bits, parity bits and additional parity bits, and fed to the turbo decoder to perform classical turbo decoding by MAP algorithm.

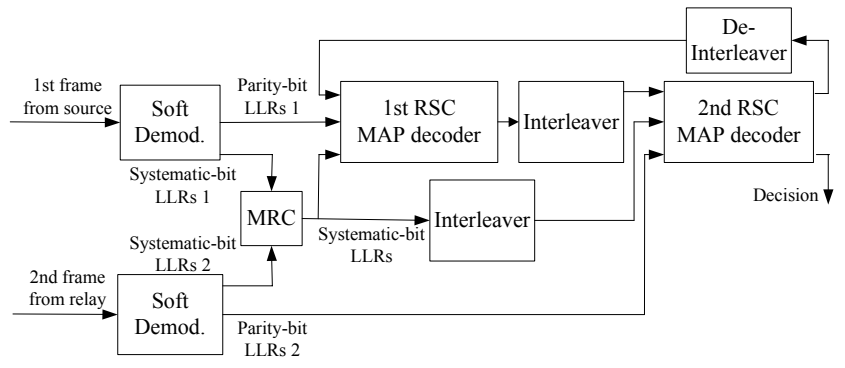

Fig. 4. The block diagram of the decoder.

The turbo decoder consists of two RSC decoders and several interleavers/de-interleavers, as shown in Fig.4. The first RSC MAP decoder produces the extrinsic information for the systematic bits, which is interleaved then passed to the second RSC MAP decoder as the a priori information for the interleaved systematic bits. The second RSC MAP decoder produces the extrinsic information for the interleaved systematic bits, which is de-interleaved and then passed to the first RSC MAP decoder as the a priori information for the systematic bits. The soft information is iteratively updated and transferred between two RSC decoders, and hard decision is made on the output of the second RSC MAP decoder after several iterations.

\section{PERFormance ANALYSIS}

In this section, we present an analytical methodology for evaluating the performance of the proposed DTC-SIR scheme. Union upper bound to bit error probability is analyzed.

For linear codes, the pairwise error probability (PEP) for a coded system is the probability that detected another erroneous codeword with Hamming weight $d$.

Firstly, we consider the conventional DTC-SIR scheme. The PEP conditioned on the various received SNR values, is given by [8]

$$
P_{1}\left(d \mid h_{\mathrm{SD}}, h_{\mathrm{RD}}, h_{\mathrm{SR}}\right)=Q\left(\sqrt{2 d_{1} \gamma_{\mathrm{SD}}+2 d_{2} \gamma_{\mathrm{RD}}}\right)
$$

where $d_{1}$ and $d_{2}$ are the number of erroneous bits in the Hamming weight $d$ that are transmitted through source-todestination channel and relay-to-destination channel, respectively. At the destination, the received signals consist of two parts: the first part is the transmitted signals from the source consisting of systematic bits and parity bits, so $d_{1}$ is the number of erroneous bits in the first coded sequence; the second part is the transmitted signals from the relay consisting of additional parity bits generated from the interleaved systematic bits, so $d_{2}$ is the number of erroneous bits in the second parity sequence.

In the proposed DTC scheme, the relay transmits not only the soft information of additional parity bits but also the soft information of systematic bits. The PEP conditioned on the received SNR values can be expressed as

$$
P_{2}\left(d \mid h_{\mathrm{SD}}, h_{\mathrm{RD}}, h_{\mathrm{SR}}\right)=Q\left(\sqrt{2 d_{1}\left(\gamma_{\mathrm{SD}}+\gamma_{\mathrm{RD}}\right)+2 d_{2} \gamma_{\mathrm{RD}}}\right)
$$

where $d_{1}$ is the number of the erroneous bits in the systematic and the parity bits, $d_{2}$ is the number of the erroneous bits in the additional parity bits. Since the distance spectrum is inherent nature for a coding which is independent from the channel condition, $d_{1}$ and $d_{2}$ are the same as in (8). When the relay repeats the soft information of the systematic bits, different from (7), the SNR corresponding Hamming weight $d_{1}$ is the summation of $\gamma_{\mathrm{SD}}$ and $\gamma_{\mathrm{RD}}$ as in (8).

To obtain the unconditional PEP, we should make the expectation over the distributions of the fading coefficients. Let $P(d)$ be the unconditional PEP of decoding an erroneous code sequence with weight $d$, which is given by [8]

$$
\begin{aligned}
P(d)= & \int h_{\mathrm{SR}} \int_{h_{\mathrm{RD}}} \int h_{\mathrm{SD}} P\left(d \mid h_{\mathrm{SD}}, h_{\mathrm{RD}}, h_{\mathrm{SR}}\right) \\
& \times p\left(h_{\mathrm{SD}}\right) p\left(h_{\mathrm{RD}}\right) p\left(h_{\mathrm{SR}}\right) d\left(h_{\mathrm{SD}}\right) d\left(h_{\mathrm{RD}}\right) d\left(h_{\mathrm{SR}}\right)
\end{aligned}
$$

From (7) (8) (9), we can obtain the unconditional PEP of the DTC-SIR and proposed DTC scheme, denoted by $P_{1}(d)$ and $P_{2}(d)$ respectively. At a high SNR, they can be approximated as [8]

$$
\begin{gathered}
P_{1}(d) \leq \frac{1}{2}\left(\gamma_{\mathrm{SD}}\right)^{-1}\left(\frac{1}{R d_{\mathrm{free}} \gamma_{\mathrm{SR}}}+\frac{1}{\gamma_{\mathrm{RD}}}\right)\left(d_{1} d_{2}\right)^{-1} \\
P_{2}(d) \leq \frac{1}{2}\left(\gamma_{\mathrm{SD}}+\gamma_{\mathrm{RD}}\right)^{-1}\left(\frac{1}{R d_{\mathrm{free}} \gamma_{\mathrm{SR}}}+\frac{1}{\gamma_{\mathrm{RD}}}\right)\left(d_{1} d_{2}\right)^{-1}
\end{gathered}
$$

The average upper bound on the bit error rate (BER) can be expressed as [10]

$$
P_{b} \leq \sum_{z=0}^{K} \sum_{y=0}^{K} \sum_{w=1}^{K} \frac{w}{K} a_{w, z, y} P(d)
$$

where $a_{w, z, y}$ denotes the multiplicity of code words corresponding to input weight $w$ and parity weights $z$ and $y$. 
Note that $d_{1}$ is equal to the summation of the exponents of $w$ and $z$, and $d_{2}$ is equal to the exponents $y$.

From (10) and (11), it is clear that the proposed scheme outperforms the conventional DTC-SIR due to the repeated soft systematic bits by the relay.

\section{Simulation RESUlts AND Discussions}

In this section, the bit error rate (BER) performance is evaluated through simulation. For comparison purposes, we consider two reference schemes which are the traditional DTC scheme and DTC-SIR scheme. For the concerned three-node relay system, it is assumed to employ BPSK modulation scheme. The channel code at the source and the relay contains a rate $1 / 2$ RSC code with constraint length 4 and information bit block length of 96 bits. Moreover, all three nodes hold the normalized transmit power.

The SNR setting is as followed.

$$
\begin{gathered}
\gamma_{\mathrm{SR}}=\gamma_{\mathrm{SD}}+\delta \\
\gamma_{\mathrm{SD}}=\gamma_{\mathrm{RD}}
\end{gathered}
$$

Namely, the source-to-relay channel is assumed $\delta \mathrm{dB}$ better than the source-to-destination channel while the sourceto-destination channel has the same channel quality as the relay-to-destination channel. Three cases are concerned, and the BER simulation results are depicted in Fig. 5. through Fig. 7 for $\delta=10,15$ and $20 \mathrm{~dB}$. From the simulation results, we observe that the proposed scheme outperforms the other two schemes in all cases. For example, at the BER level of $10^{-3}$, the SNR gain of the proposed scheme is about $2 \mathrm{~dB}$ and $0.5 \mathrm{~dB}$ compared to the traditional DTC scheme and the DTC-SIR scheme in case of $\delta=10 \mathrm{~dB}$.

We can obtain two observations from the simulation results. Firstly, it is advantageous to transmit soft estimates due to the mitigation of the error propagation. Secondly, it is also advantageous to repeat soft systematic bits in the second frame to achieve diversity gain. The combination of these two ideas can improve the overall performance.

\section{CONCLUSION}

In this paper, we proposed an enhanced distributed turbo code scheme with soft systematic bit repeating for three-node relay-enhanced wireless communication systems, targeting to improve error performance by mitigating the decoding error propagation and gaining diversity. For the proposed scheme, the relay transmits the soft information of both the additional parity bits and the systematic bits. To avoid the loss of spectrum efficiency due to the repetition of systematic information, parts of the soft information generated at the relay are punctured before forwarding. Both the theoretical analysis and numerical simulation verify that the proposed scheme effectively improves the error performance of the relay system in quasi-static fading channel.

\section{ACHNOWLEDGEMENTS}

This work was supported by National Key Technology R\&D Program of China under Grant 2009ZX03005-003-02 and Fundamental Research Funds for Central Universities under Grant 2009RC0102.

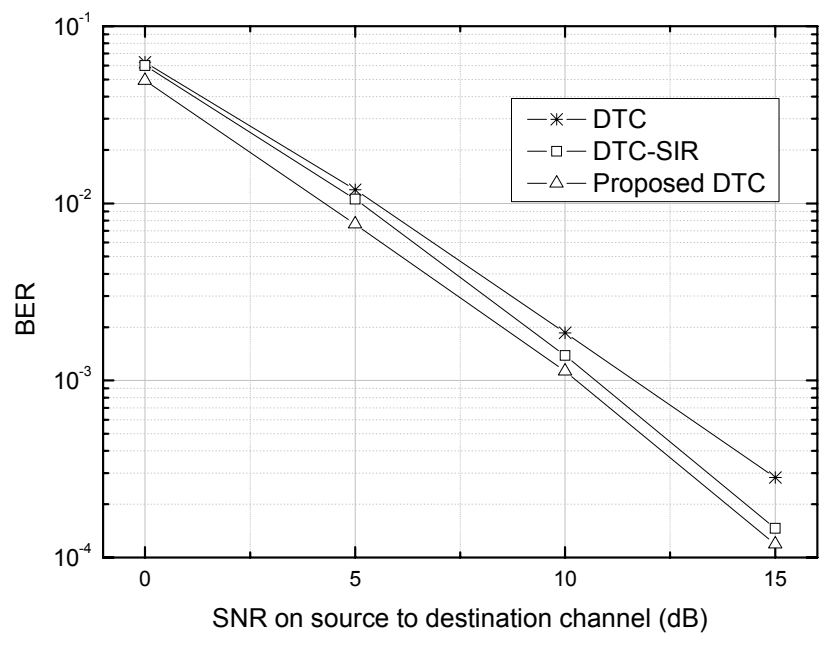

Fig. 5. BER performance comparison when $\delta=10 \mathrm{~dB}$.

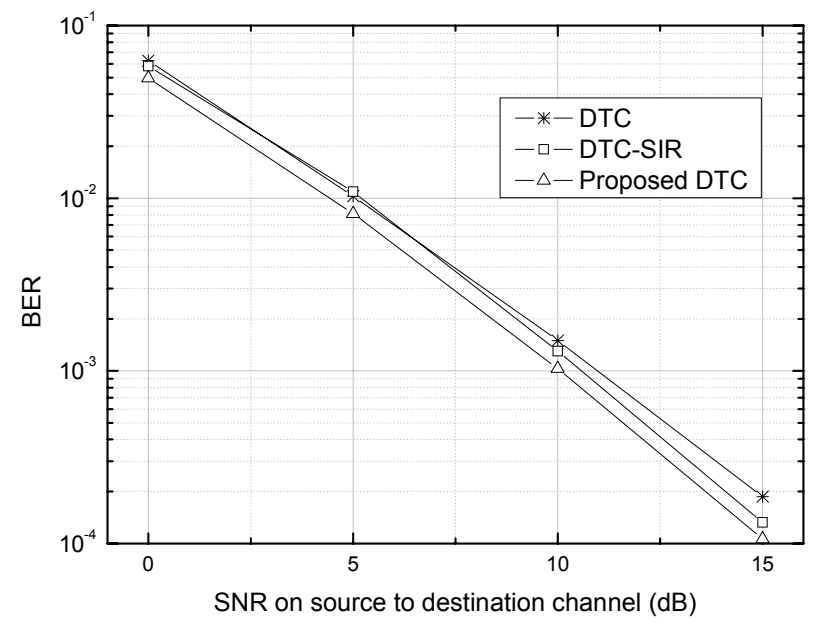

Fig. 6. BER performance comparison when $\delta=15 \mathrm{~dB}$.

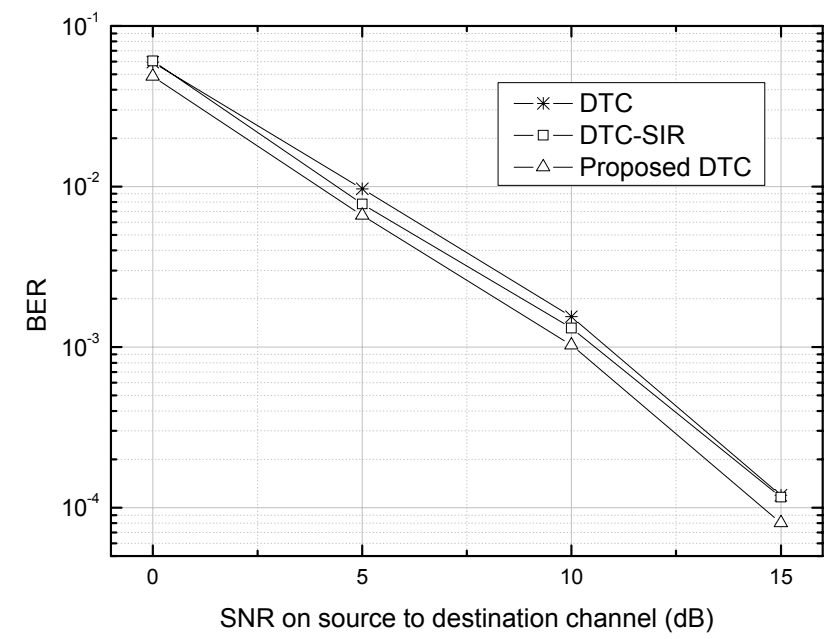

Fig. 7. BER performance comparison when $\delta=20 \mathrm{~dB}$

\section{REFERENCES}


[1] E. C. van der Meulen, "Three-terminal communication channels," Adv. Appl. Prob., vol. 3, pp. 120-154, 1971.

[2] N. Laneman, "Cooperative diversity in wireless networks: algorithms and architectures," Ph.D. dissertation, Massachusetts Institute of Technology, Cambridge, MA, Aug. 2002.

[3] X. Bao and J. Li, "Efficient Messages Relaying for Wireless User Cooperation: Decode-Amplify-Forward (DAF) and Hybrid DAF and Coded-Cooperation," IEEE Trans. on Wireless Commun.,vol.6, no.11,pp. 3975-3984, Nov 2007.

[4] J. N. Laneman and G. W. Wornell, "Distributed space-time-coded protocols for exploiting cooperative diversity in wireless networks," IEEE Trans. Inf. Theory, vol. 49, pp. 2415-2425, Oct. 2003.

[5] M. Dohler, M. Hussain, A. Desai, and H. Aghvami, "Performance of distributed space-time block codes," in Proc. IEEE VTC-Spring, May 2004, vol. 2, pp. 742-746.

[6] M. Janani, A. Hedayat, T. E. Hunter, A. Nosratinia, "Coded cooperation in wireless communications: space-time transmission and iterative decoding," IEEE Trans. Signal Process., vol. 52,,no.2, pp. 362-371, Feb. 2004.

[7] B. Zhao and M. C. Valenti, "Distributed turbo codes: Towards the capacity of the relay channel," in Proc. VTC-Fall, Oct. 2003, vol. 1, pp.322-326.

[8] Y. Li, B. Vucetic, T. Wong, and M. Dohler, "Distributed turbo coding with soft information relaying in multi-hop relay networks", IEEE Journal on Selected Areas in Commun., vol.24, no.11, pp. 2040-2050, Nov.2006.

[9] H. T. Nguyen, H. H. Nguyen, and T. Le-Ngoc, "A bandwidth-efficient coded cooperative communications system," in Proc. IEEE VTC-Fall, Sept.2006. 